\title{
EFFECT OF PROCESS VARIABLES USING SQUARE TYPE CUTTING TOOL ON SURFACE ROUGHNESS AND RESIDUAL STRESS OF EN24 IN AN END-MILLING PROCESS
}

\author{
VISHAL SINGH $^{1}$, SHIKHAR ZUTSHI ${ }^{2}$, UTKARSH GOEL ${ }^{3}$ \& QASIM MURTAZA $^{4}$ \\ ${ }^{1,2,3}$ Delhi Technological University (Formerly Delhi College of Engineering), Delhi, India \\ ${ }^{4}$ Professor, Department of Mechanical Engineering, Delhi Technological University, Delhi, India
}

\begin{abstract}
CNC machining has been one of the most reliable, versatile and efficient manufacturing methods having precise production capacity. In order to design and produce complicated surfaces, surface characteristics and cutting parameters play a crucial role. The goal of this paper is to study the effect of 5 different levels of process variables (spindle speed, feed rate and depth of cut) using square type cutting tool on surface roughness and residual stress of EN24 in an end-milling machining process. The prediction of surface roughness and residual stress is done by developing a mathematical model on the basis of RSM (Response Surface Methodology) and ANOVA (Analysis Of Variance). Results show that minimum surface roughness and residual stress is obtained at minimum feed rate, minimum depth of cut and medium spindle speed. KEYWORDS: EN24, End-Milling, Surface Roughness, Residual Stress, RSM, Optimization \& ANOVA
\end{abstract}

Received: Mar 21, 2020; Accepted: Apr 11, 2020; Published: Apr 28, 2020; Paper Id.: IJMPERDJUN202014

\section{INTRODUCTION}

The millennial metal industry is focusing on tackling the challenges of boosting the productivity and quality of final machined components. A significant effort to study all aspects of different machining parameters can be observed around the world. The process of end milling is used as a finishing step post rough machining in almost all milled components. The importance of this process is increasing exponentially with the technological advancements; and innovations in the modern era call for further efficient and economical machining outcomes, in which surface finish, hardness and residual stress remain some of the most critical factors.

Being a moderate carbon in addition to low-alloy steel, typical applications of EN24 include machined tool parts majorly in the automobile industry. Owing to strain-hardening as well as diffusing tendencies between tool and work material and low specific heat, this alloy falls in the category of hard to machine materials. High Cutting tool temperatures, built up edge formation, considerable cutting forces along with undesirable surface finish are the common problems experienced in the attempt of machining this alloy. Surface characteristics have the potential to influence factors like corrosion resistance, surface friction, fatigue strength, load bearing capacity, creep life, lubricant holding capacity etc. A huge consideration was made while performing the experiment for this research to keep the environmental conditions same while measuring all the parameters of Surface finish, and residual stress both before and after the machining process so that accurate and reliable results are obtained and significant conclusions could be drawn without much errors.

Mathematical models as well as statistical Design of Experiments (DOE) have been extensively used to 
determine the surface quality. Design of experiments deals with strategic planning, examining and deciphering controlled tests to assess the components bringing about substantial and targeted results. Tseng et al. [1] discussed two different approaches to improve quality control of metal cutting processes. Design of Experiments (DOE) was used to determine the significance of factors followed by an approach including fuzzy logic was presented for the prediction of surface roughness. The factors in consideration for DOE in the experiment were feed rate per tooth, depth of cut, tool nose radius, cutting speed, the use of cutting fluid and the three components of the cutting force.

Masmiati et al. [2] using inclined end milling cutting parameters optimized the cutting to obtain efficient surface integrity in view of micro hardness and residual stress, designing the experimental work using Taguchi optimization, while data analysis being conducted search the optimal conditions using $\mathrm{S} / \mathrm{N}$ (signal to-noise ratio) response analysis as well as PARETO ANOVA (analysis of variance). It was found that Surface inclination angle and micro hardness are directly proportional, however residual stress was found to be inversely proportional.

Bhattacharya et al. [3] carried out high speed machining of AISI 1045 steel. Analysis was done using ANOVA and it was found that the most significant input factor was cutting speed. Ozel et al. [4] attempted a hard turning machining of AISI H13 steel and tried analysing the different forces and roughness associated with the machining using ANOVA. Effect of feed rate, cutting speed and DOC on surface roughness and residual stress in turning process of Inconel 718 super alloy was studied by Farshid et al. [5]. Responses were optimized with the help of hybrid ANN-GA technique and optimal machining parameters were found.

Saikumar and Shunmugam [6] investigated high-speed (rough cut \& finish cut) end-milling cutting on EN24 (AISI 4340) steel of hardness $45 \mathrm{HRc}$ with TiAlN-coated insert of an integrated framework. For rough cut of end-milling, measuring of tool wear, material removal volume and cutting forces was done. Similarly, prediction of a set of cutting parameters was done on DOE for a given interval of surface roughness with the help of a response surface model.

Gupta et al. [7] presented process parameters effects like feed rate, cutting speed and various cooling situations on tool wear in operations on EN24 alloy steel while an uncoated Ti-Carbide insert tool was used. As per Taguchi's L9 array (orthogonal) experiments were carried out and statistical ANOVA (Analysis Of Variance) was used to find the effect of parameters on responses on the basis of their P-value and F-value (95\% confidence level). Overall, it was observed that cryogenic cooling can provide an improved tool life which in turn results in an extended product life much better performance.

From these references, it is evident that relatively limited work of end milling on EN-24 has been done, which optimizes surface roughness and residual stress. Thus, after extensive consideration and discussion, this combination was chosen to be the major objective of this research.

\section{MATERIALS AND METHODS}

\subsection{Workpiece Material}

EN24 is most suitable for components having large cross section subjected to high stress owing to its high strength and excellent machinability. It is used for manufacturing of aircraft components, heavy duty axles, gears, bolts, studs etc. It can be further surface hardened to improve wear resistance. The chemical composition of EN24 is given in Table 1 [6][8]. 
Table 1: Chemical Composition of EN24

\begin{tabular}{|l|c|}
\hline \multicolumn{1}{|c|}{ Element } & Range \\
\hline Carbon $(\mathrm{C})$ & $0.37-0.44$ \\
\hline Nickel (Ni) & $1.55-2.00$ \\
\hline Silicon (Si) & $0.10-0.35$ \\
\hline Manganese (Mn) & $0.50-0.90$ \\
\hline Chromium (Cr) & $0.65-0.95$ \\
\hline Sulphur (S) & $<0.04$ \\
\hline Molybdenum (Mo) & $0.20-0.35$ \\
\hline Phosphorous (P) & $<0.04$ \\
\hline Iron (Fe) & Remainder \\
\hline
\end{tabular}

\subsection{Tool Material}

Tool used was SGS 48509 Solid carbide tool having coating of Titanium. It is a multi-layered structure tool with hardness up to $2200 \mathrm{HV}$ having diameter of $6 \mathrm{~mm}$. This type of tool is broadly used in aerospace, automotive, casting \& foundries, general engineering purpose, etc.

\subsection{Process Variables and Limits}

Feed rate, depth of cut and spindle speed are being scrutinized as process variables. Each factor is varied at five levels as given in Table 2 and the design is prepared using Stat-Ease Design Expert Version 11.

Table 2: Various Levels of Each Variable

\begin{tabular}{|l|c|c|c|c|c|}
\hline \multirow{2}{*}{\multicolumn{1}{|c|}{ Variable }} & \multicolumn{5}{|c|}{ Levels } \\
\cline { 2 - 6 } & $\mathbf{1}^{\text {st }}$ & $\mathbf{2}^{\text {nd }}$ & $\mathbf{3}^{\text {rd }}$ & $\mathbf{4}^{\text {th }}$ & $\mathbf{5}^{\text {th }}$ \\
\hline Spindle Speed (RPM) & 500 & 1000 & 1500 & 3000 & 3500 \\
\hline Feed rate (mm / rev) & 600 & 1200 & 1800 & 2400 & 3000 \\
\hline Depth of cut (mm) & 0.1 & 0.2 & 0.3 & 0.4 & 0.5 \\
\hline
\end{tabular}

\subsection{Experiment Details}

The machining was carried out on the specimen material having dimensions $(10 \mathrm{~cm} \mathrm{X} 5 \mathrm{~cm} \mathrm{X} 2 \mathrm{~cm})$ with the help of a solid carbide tool (SGS) 48509. Siemens Sinumerik 828D CNC milling machine was used to perform milling. Siemens Sinumerik 828D have rugged hardware architecture and intelligent control algorithms along with premium drive and motor technology, thereby, providing high precision machining. It has the maximum spindle speed of 20000 RPM. Coolant was used during machining. Specimens were marked from 1 to 20 for each experimental run. A thin layer was machined from all specimens before conducting machining tests. Fresh tool was used for machining so as to avoid the effect of tool wear on experimental results.

Closely spaced surface irregularities are termed as surface roughness. It is measured using Taylor Hobson Surtronic $3+$ profiler and along the direction of length of cut. Roughness measurements on the work pieces have been repeated three times and average of these values have been reported in the results. A $\mu$-X360 Portable X-ray Residual Stress Analyser was used to measure residual stresses. From a single incident X-ray angle, it can detect full Debye ring making it capable of evaluating stress effectively. Parameters for residual stress measurement include x-ray irradiation time (Setup + Measurement) $60 \mathrm{sec}, 0.280$ Poisson's ratio, Young's modulus of $224 \mathrm{GPa}$.

\section{RESULTS AND DISCUSSIONS}

Response values i.e. values of surface roughness and residual stress are summarized in Table 3 . 
Table 3: Experimental Design with Response

\begin{tabular}{|c|c|c|c|c|c|}
\hline Run no & $\begin{array}{c}\text { Spindle } \\
\text { Speed (RPM) }\end{array}$ & $\begin{array}{c}\text { Depth of } \\
\text { Cut }(\mathbf{m m})\end{array}$ & $\begin{array}{c}\text { Feed rate } \\
(\mathbf{m m} / \mathbf{m i n})\end{array}$ & $\begin{array}{c}\text { Surface } \\
\text { Roughness }(\boldsymbol{\mu m})\end{array}$ & $\begin{array}{c}\text { Residual } \\
\text { Stress }(\mathbf{M P a})\end{array}$ \\
\hline 1 & 1500 & 0.3 & 1800 & 2.11 & 380 \\
\hline 2 & 3000 & 0.1 & 1200 & 1.88 & 356 \\
\hline 3 & 1500 & 0.5 & 600 & 1.88 & 295 \\
\hline 4 & 3500 & 0.1 & 2400 & 2.28 & 405 \\
\hline 5 & 3000 & 0.3 & 1800 & 2.18 & 360 \\
\hline 6 & 3500 & 0.3 & 600 & 1.78 & 358 \\
\hline 7 & 1500 & 0.1 & 600 & 1.4 & 300 \\
\hline 8 & 3500 & 0.5 & 1200 & 2.16 & 398 \\
\hline 9 & 1500 & 0.3 & 1800 & 2.06 & 413 \\
\hline 10 & 3000 & 0.4 & 3000 & 3.09 & 526 \\
\hline 11 & 500 & 0.3 & 600 & 2.94 & 204 \\
\hline 12 & 500 & 0.5 & 1800 & 3.0 & 290 \\
\hline 13 & 1500 & 0.5 & 600 & 1.48 & 430 \\
\hline 14 & 1000 & 0.5 & 3000 & 3.6 & 368 \\
\hline 15 & 1500 & 0.3 & 1800 & 2.2 & 440 \\
\hline 16 & 500 & 0.3 & 3000 & 4.16 & 260 \\
\hline 17 & 3000 & 0.4 & 3000 & 3.36 & 550 \\
\hline 18 & 1500 & 0.3 & 1800 & 2.1 & 340 \\
\hline 19 & 500 & 0.1 & 1800 & 3.14 & 145 \\
\hline 20 & 1000 & 0.1 & 3000 & 3.3 & 280 \\
\hline
\end{tabular}

\subsection{Surface Roughness}

Quadratic model for surface roughness is chosen as it does not contain any aliased terms. ANOVA for this is shown in Table 4. Only significant terms are shown in the table 4 . The minimum value of the response is $1.4 \mu \mathrm{m}$ and maximum value is $4.16 \mu \mathrm{m}$.

Table 4: ANOVA for Quadratic Model for Surface Roughness

\begin{tabular}{|c|c|c|c|c|c|c|}
\hline Source & Sum of Squares & df & Mean Square & F-value & p-value & \\
\hline Model & 10.49 & 9 & 1.17 & 25.81 & $<0.0001$ & significant \\
\hline A-Spindle Speed & 1.57 & 1 & 1.57 & 34.73 & 0.0002 & \\
\hline B-Feed & 4.96 & 1 & 4.96 & 109.81 & $<0.0001$ & \\
\hline $\mathrm{A}^{\mathbf{2}}$ & 1.91 & 1 & 1.91 & 42.39 & $<0.0001$ & \\
\hline $\mathrm{B}^{2}$ & 0.3007 & 1 & 0.3007 & 6.66 & 0.0274 & \\
\hline Residual & 0.4517 & 10 & 0.0452 & & & \\
\hline Lack of Fit & 0.3248 & 5 & 0.0650 & 2.56 & 0.1628 & not significant \\
\hline Pure Error & 0.1269 & 5 & 0.0254 & & & \\
\hline Cor Total & 10.95 & 19 & & & & \\
\hline
\end{tabular}

Model terms having P-values less than 0.05 are considered as significant whereas on the other hand those having P-values more than 0.10 are not significant. In our investigation $\mathrm{A}, \mathrm{B}, \mathrm{A}^{2}, \mathrm{~B}^{2}$ are significant model terms. High Model Fvalue i.e. 25.81 suggests that the model is significant. There is a very little chance that this F-value could be due to noise. Fit statistics is shown in Table 5. Non-significant lack of fit with F-value of 2.56 having only $16.28 \%$ possibilities could be due to the noise shows that this model can be used with good accuracy.

Table 5: Fit Statistics

\begin{tabular}{|c|c|c|c|c|c|c|}
\hline Std. Dev. & Mean & C.V. \% & $\mathbf{R}^{\mathbf{2}}$ & Adjusted R $^{\mathbf{2}}$ & Predicted R $^{\mathbf{2}}$ & Adequate Precision \\
\hline 0.2125 & 2.50 & 8.48 & 0.9587 & 0.9216 & 0.7496 & 17.4613 \\
\hline
\end{tabular}


$\mathrm{R}^{2}$ which is known as the coefficient of determination is found to be 0.9587 indicating the $95.87 \%$ of variability in response, i.e. surface roughness can be justified by the model. The difference between predicted $\mathrm{R}^{2}$ and adjustedR is less than 0.2 which shows that they are in practical correspondence with each other. Signal to noise ratio is shown by Adequate Precision where more than 4 is good. Adequate precision of 17.461 indicates an adequate signal which shows that this model can be used to traverse the design space.

\subsection{Final Equation}

Surface Roughness $=+1.94-0.3941 \mathrm{~A}+0.7125 \mathrm{~B}+0.1242 \mathrm{C}+0.0478 \mathrm{AB}+0.1082 \mathrm{AC}+0.0931 \mathrm{BC}+0.8572 \mathrm{~A}^{2}+0.2720 \mathrm{~B}^{2}-$ $0.1006 C^{2}$

Equation 1 is in terms of coded factors. +1 is considered as high level and -1 as low level in coded equation. Relative impact of factors can be identified easily from coded equation but for making predictions about the output at particular levels actual equation has to be used.

\section{Surface Roughness \\ Color points by value of Surface Roughness: \\ 1.4 \\ 4.16}

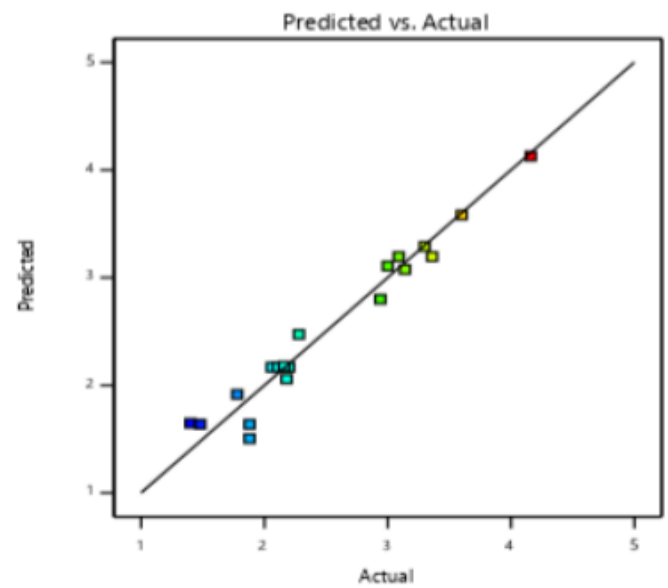

Graph 1: Predicted vs Actual value of Surface Roughness.

Graph 1 shows the relationship between predicted values by the model and the experimental values. These values are very well in correlation with each other.

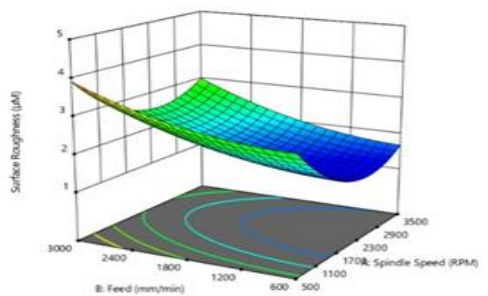

(a)

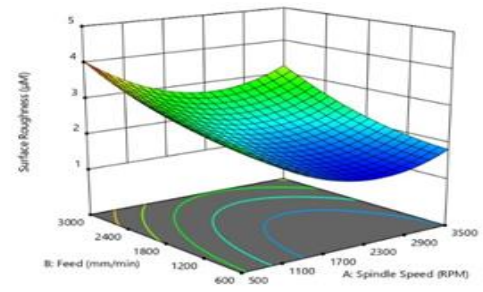

(b)

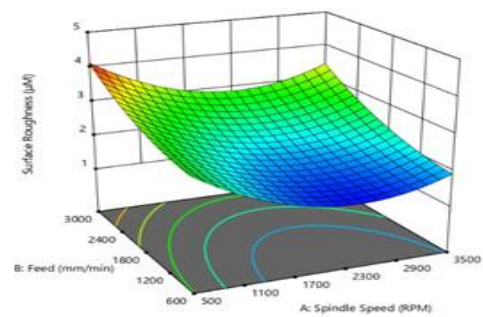

(c)

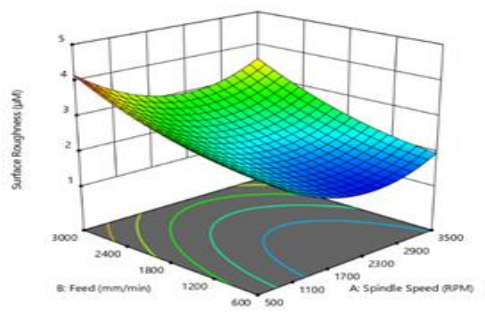

(d)

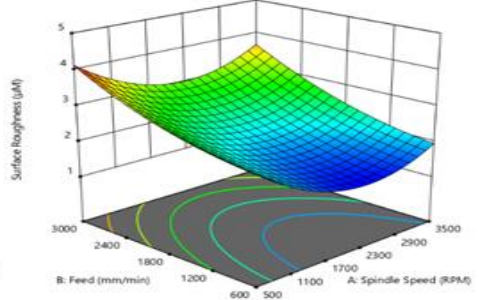

(e)

Figure 1: Contour Plots for Surface Roughness at different Values of Depth of Cut. 
At first depth of cut was kept constant (at different levels of 0.1, 0.2,0.3,0.4, $0.5 \mathrm{~mm}$ ) and effects of spindle speed and feed rates $(\mathrm{mm} / \mathrm{min})$ were studied and shown in Figure.1. Surface roughness was found be at its lowest at spindle speed more than 1700 RPM and feed rate less than $1200 \mathrm{~mm} / \mathrm{min}$. The effect of feed rate and spindle speed is less significant with the depth of cut, but it was observed that by increasing the feed rate and depth of cut and by decreasing the spindle speed, surface roughness increases. Material removal will increase with the increase in depth of cut thereby resulting in increase in peaks of cut portion.

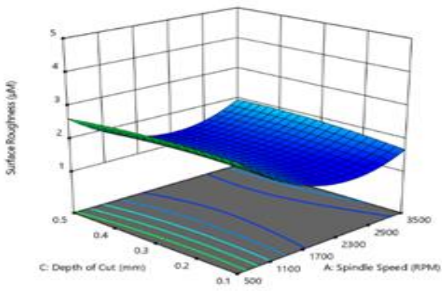

(a)

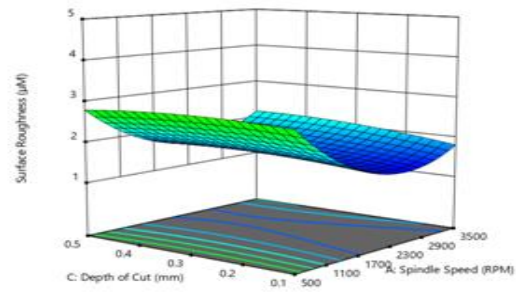

(b)

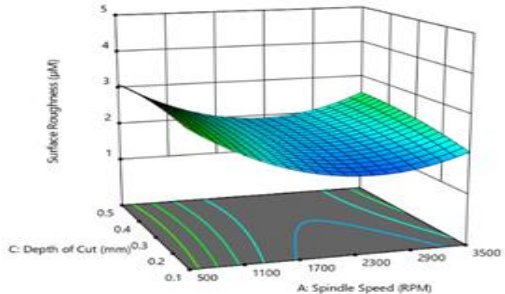

(c)

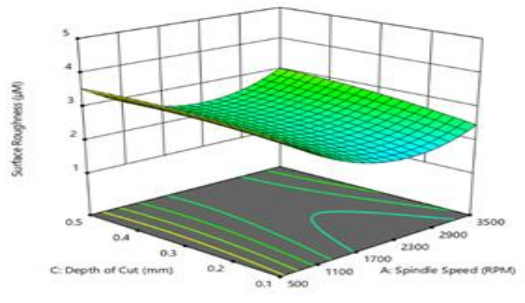

(d)

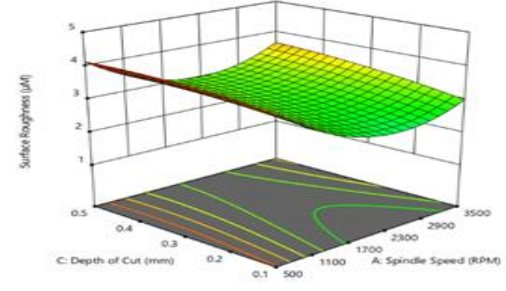

(e)

Figure 2: Contour Plots for Surface Roughness at different value of Feed Rate.

In the next stage, feed rate was kept constant (at different levels of $600,1200,1800,2400,3000 \mathrm{~mm} / \mathrm{min}$ ) and effects of depth of cut and spindle speed were studied on the surface roughness and shown in Figure. 2. It can be observed that as the feed rate increases from $600 \mathrm{~mm} / \mathrm{min}$ to $3000 \mathrm{~mm} / \mathrm{min}$, value of surface roughness increases. Also, in the medium range of Spindle Speed, minimum value of surface roughness is obtained at constant feed rate whereas moderate to high value of surface roughness is obtained at low and high Spindle SPEED.

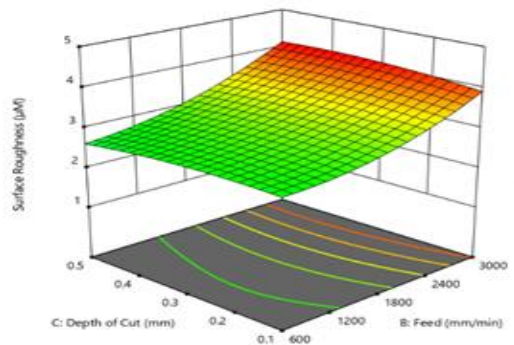

(a)

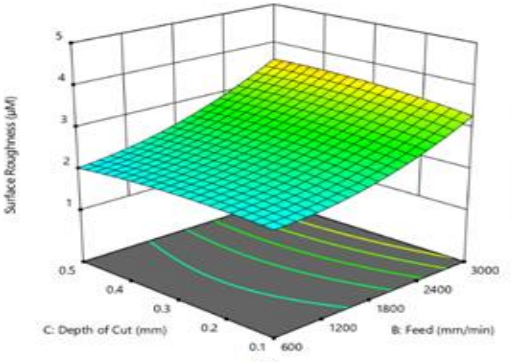

(b)

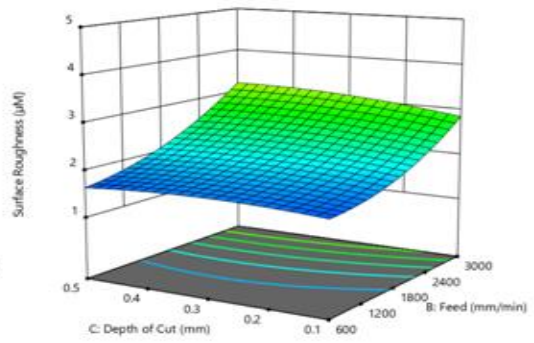

(c)

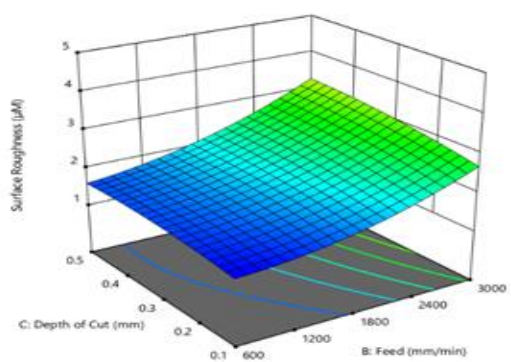

(d)

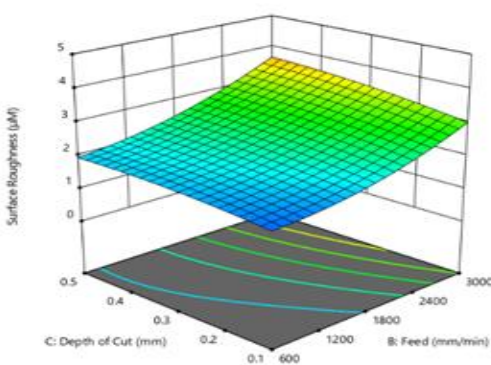

(e)

Figure 3: Contour Plots for Surface Roughness at different values of Spindle Speed. 
In the last stage, spindle speed was maintained constant (at different levels of 500, 1000, 1500, 3000, 3500 RPM) and the effects of feed rate and depth of cut on surface roughness were studied and shown in Figure.3. Lower the Spindle speed and higher the feed rate, higher will be the surface roughness. When the spindle speed will increase, then time of contact between chip and surface will be lesser thereby decreasing the deterioration effect of chips and hence improving surface roughness.

\subsection{Residual Stress}

ANOVA for quadratic model for residual stress is shown in Table 6. Non-significant terms is removed and the minimum value for response is $145 \mathrm{MPa}$ and maximum value is $550 \mathrm{MPa}$.

High model F-value of 7.29 suggests that the model is significant. There are very low possibilities that this F-value could be due to noise. In this case A, B, C, $\mathrm{A}^{2}$ are significant model terms.

Fit statistics are shown in Table 7. Non-significant lack of fit with F-value of 0.60 having only $70.57 \%$ possibilities that this could be due to the noise shows that this model can be used with good accuracy.

Table 6: ANOVA for Quadratic Model for Surface Roughness

\begin{tabular}{|c|c|c|c|c|c|c|}
\hline Source & Sum of Squares & df & Mean Square & F-value & p-value & \\
\hline Model & $1.573 \mathrm{E}+05$ & 9 & 17478.48 & 7.29 & 0.0023 & significant \\
\hline A-Spindle Speed & 82466.48 & 1 & 82466.48 & 34.39 & 0.0002 & \\
\hline B-Feed & 22673.77 & 1 & 22673.77 & 9.46 & 0.0117 & \\
\hline C-Depth of Cut & 17483.05 & 1 & 17483.05 & 7.29 & 0.0223 & \\
\hline $\mathrm{A}^{2}$ & 25998.33 & 1 & 25998.33 & 10.84 & 0.0081 & \\
\hline Residual & 23977.49 & 10 & 2397.75 & & & \\
\hline Lack of Fit & 8990.24 & 5 & 1798.05 & 0.5999 & 0.7057 & not significant \\
\hline Pure Error & 14987.25 & 5 & 2997.45 & & & \\
\hline Cor Total & $1.813 \mathrm{E}+05$ & 19 & & & & \\
\hline
\end{tabular}

Table 7: Fit Statistics

\begin{tabular}{|c|c|c|c|c|c|c|}
\hline Std. Dev. & Mean & C.V. \% & $\mathbf{R}^{\mathbf{2}}$ & Adjusted R $^{\mathbf{2}}$ & Predicted R $^{\mathbf{2}}$ & Adequate Precision \\
\hline 48.97 & 354.90 & 13.80 & 0.8677 & 0.7487 & 0.6216 & 10.6030 \\
\hline
\end{tabular}

$\mathrm{R}^{2}$ is found to be 0.8677 indicating $86.77 \%$ of variability in response i.e. residual stress which can be justified by the model. Owing to the fact that the difference is less than 0.2, the Predicted $\mathrm{R}^{2}$ of 0.6216 and Adjusted $\mathrm{R}^{2}$ of 0.7487 are in reasonable agreement i.e. Adequate Precision of 10.603 indicates that this model can be used for traversing the design space.

\subsection{Final Equation}

Residual Stress $=+420.61-90.35 \mathrm{~A}+48.17 \mathrm{~B}+43.17 \mathrm{C}+26.78 \mathrm{AB}-10.85 \mathrm{AC}+7.05 \mathrm{BC}-99.87 \mathrm{~A}^{2}+6.98 \mathrm{~B}^{2}-16.72 \mathrm{C}^{2}$

Equation 2 is in terms of coded factors. +1 is considered as high level and -1 as low level in coded equation. Relative impact of factors can be identified easily from coded equation but for making predictions about the output at particular levels actual equation has to be used. 


\section{Residual Stress}

Color points by value of Residual Stress:

145

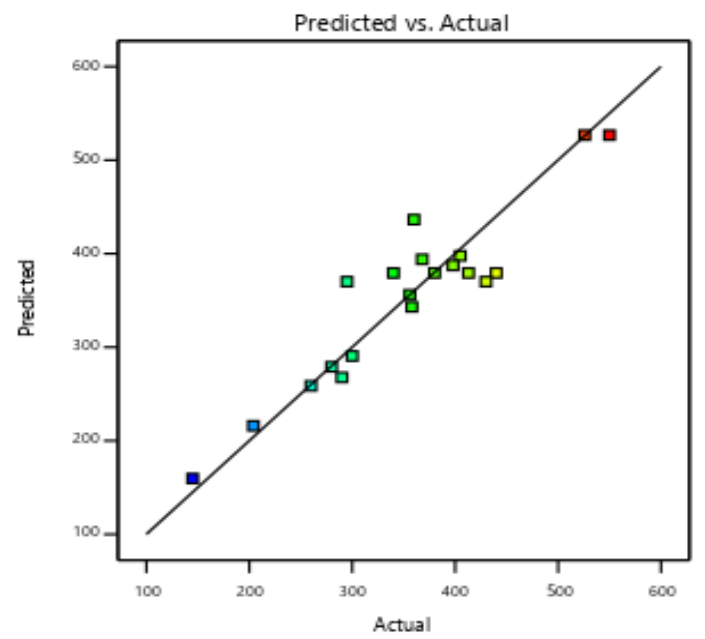

Graph 2: Predicted vs Actual value of Residual Stress.

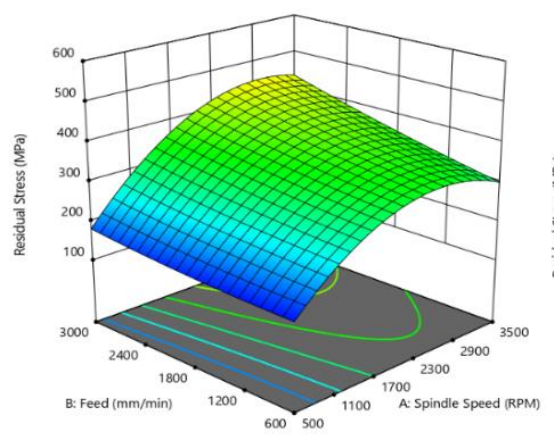

(a)

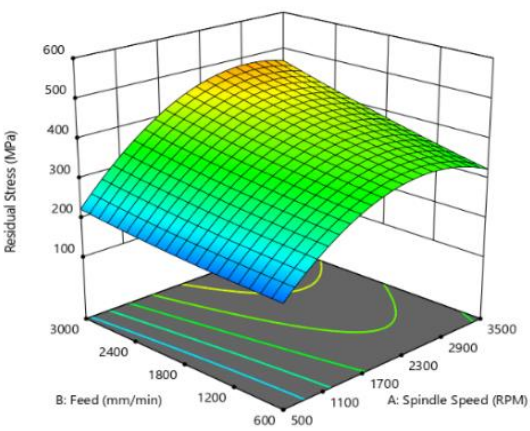

(b)

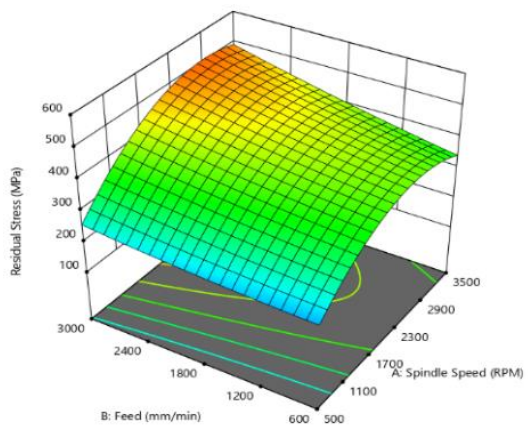

(c)

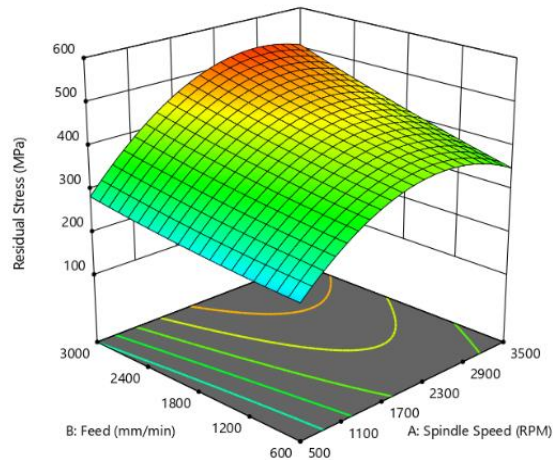

(d)

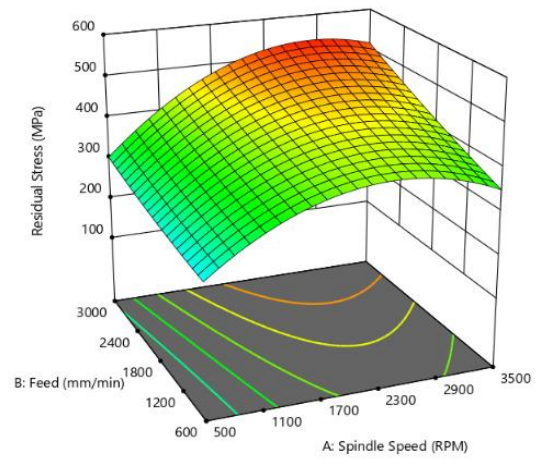

(e)

Figure 4: Contour Plots for Residual Stress at different values of Depth of Cut.

Figure.4 represents Contour plots for residual stress keeping depth of cut as constant $(0.1,0.2,0.3,0.4,0.5 \mathrm{~mm})$. With the increase in the depth of cut i.e from $0.1 \mathrm{~mm}$ to $0.5 \mathrm{~mm}$ residual stress increases. 


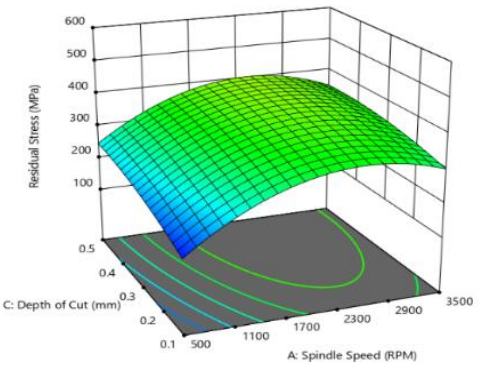

(a)

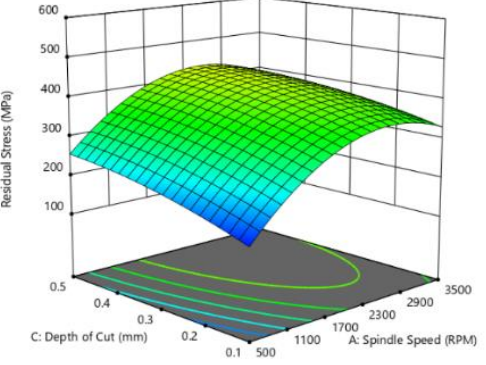

(b)

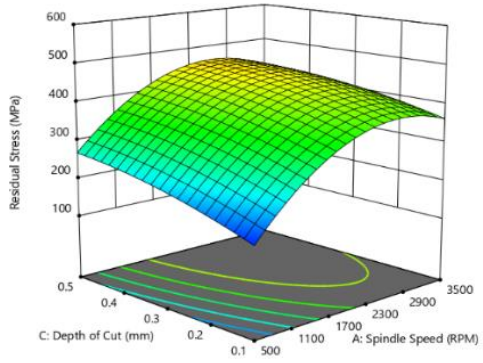

(c)

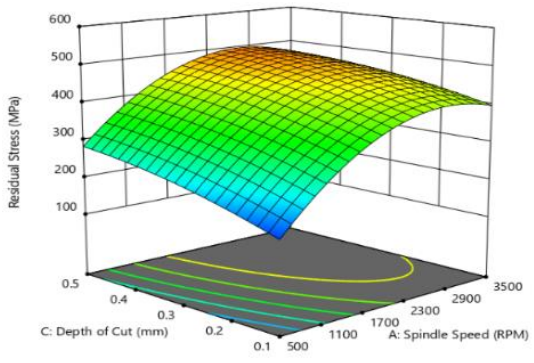

(d)

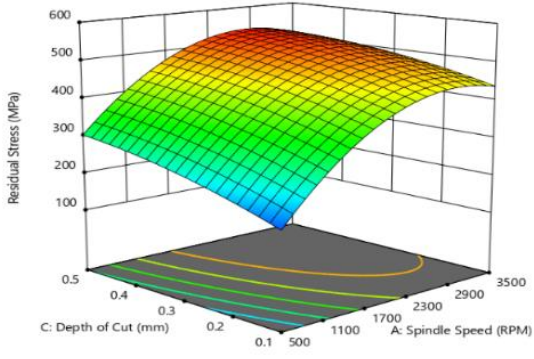

(e)

Figure 5: Contour Plots for Residual Stress at different values of Feed Rate.

Figure. 5 represents Contour Plots for residual stress keeping feed rate as constant (600, 1200, 1800, 2400, 3000 $\mathrm{mm} / \mathrm{min}$.). With the increase in feed rate, cutting forces increases as well as friction at workpiece-tool interface increases thereby resulting in the increase in residual stress.

Figure. 6 represents Contour Plots for residual stress keeping spindle speed as constant (500, 1000, 1500, 3000, 3500 RPM). At the middle range of spindle speed, it is observed that residual stress increases. As the spindle speed increases, the heat which is developed at shear zone cannot be conducted away easily as very less amount of time is available for this heat to pass from this zone and so the temperature rise results in domination of thermal effects and thereby resulting in increase residual stress.

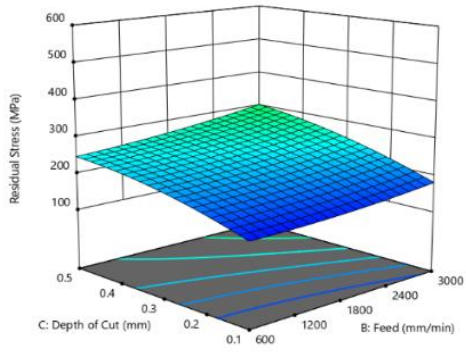

(a)

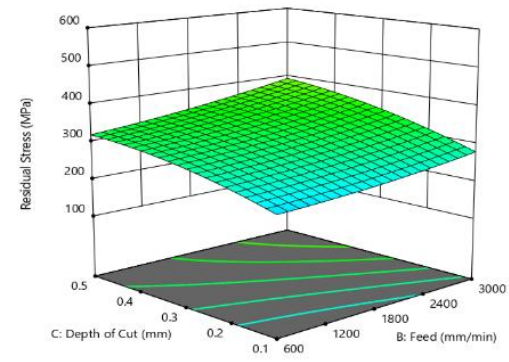

(b)

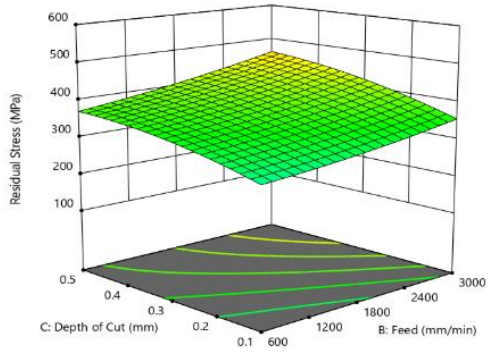

(c)

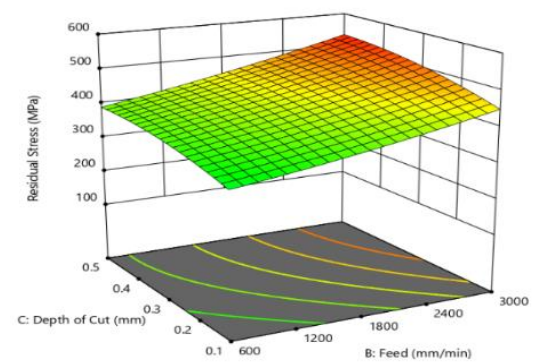

(d)

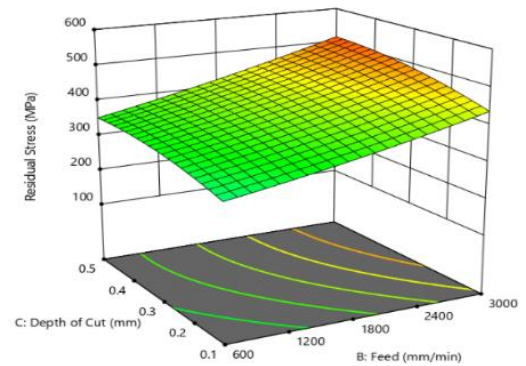

(e)

Figure 6: Contour Plots for Residual Stress at different values of Spindle Speed. 


\section{OPTIMIZATION AND CONFIRMATION}

Best results for individual responses are shown in Table 8.

Table 8: Results for Individual Response

\begin{tabular}{|c|c|c|c|c|c|}
\hline $\begin{array}{c}\text { Run } \\
\text { no }\end{array}$ & $\begin{array}{c}\text { Spindle speed } \\
(\text { RPM) }\end{array}$ & $\begin{array}{c}\text { Feed rate } \\
(\mathbf{m m} / \mathbf{m i n})\end{array}$ & $\begin{array}{c}\text { Depth of cut } \\
(\mathbf{m m})\end{array}$ & $\begin{array}{c}\text { Residual Stress } \\
(\mathbf{M P a})\end{array}$ & $\begin{array}{c}\text { Surface } \\
\text { Roughness }(\boldsymbol{\mu m})\end{array}$ \\
\hline 7 & 1500 & 600 & 0.1 & 300 & 1.4 \\
\hline 19 & 500 & 1800 & 0.1 & 145 & 3.14 \\
\hline
\end{tabular}

In Table 8, experimental run 7 gives the finest result for Surface roughness $(1.4 \mu \mathrm{m})$ but not for the Residual Stress (300 MPa). Similarly, experimental run 19 gives the finest result for Residual Stress (145 MPa) but not for the Surface Roughness $(3.14 \mu \mathrm{m})$.

Therefore, to find the optimal conditions taking into consideration all responses, desirability analysis was performed.Numerical optiization was performed on Design Expert in order to find the factors settings satisfying the defined goals as it helps in finding a point that maximizes the desirability function. The goals are shown in Table 9. Goals for response is set as minimize which implies that lower limit will be the acceptable and optimal result whereas upper limit will be the maximum possible output.

Table 9: Specified Goals for Factors and Response

\begin{tabular}{|l|l|c|c|}
\hline \multicolumn{1}{|c|}{ Factors and Response } & \multicolumn{1}{c|}{ Goal } & Upper Limit & Lower Limit \\
\hline Spindle Speed (RPM) & Within specified limits & 3500 & 500 \\
\hline Feed rate $(\mathrm{mm} / \mathrm{min})$ & Within specified limits & 3000 & 600 \\
\hline Depth of Cut $(\mathrm{mm})$ & Within specified limits & 0.5 & 0.1 \\
\hline Residual Stress $(\mathrm{MPa})$ & Minimize & 550 & 145 \\
\hline Surface Roughness $(\mu \mathrm{m})$ & Minimize & 4.16 & 1.4 \\
\hline
\end{tabular}

Desirability is elucidated when numericaly optimizing of objective function is taking place. GM (geometric mean) of individual desirabilities (di) ranging from 0 (least) to 1 (most) is known as Overall desirability (D) and is shown as:

$$
D=\left(d_{1} \cdot d_{2} \cdot \ldots \cdot d_{n}\right)^{\frac{1}{n}}=\left(\prod_{i=1}^{n} d_{i}\right)^{\frac{1}{n}} \quad \mathrm{n}=\text { the number of responses }
$$

$\mathrm{D}=0$ if any of the responses or factors fall outside their desirability range [9]. Highest desirability value was found to be 0.767 and the best parameter combination is shown in Table 10. Desirability value of 0.767 shows that minimum response can be obtained if the experiment is conducted with low feed rate, low depth of cut and medium cutting speed for EN24.

Table 10: Optimal Solution

\begin{tabular}{|c|c|c|c|c|c|c|}
\hline Number & Spindle Speed & Feed rate & Depth of Cut & Residual Stress & Surface Roughness & Desirability \\
\hline 1 & 1254.173 & 600.006 & 0.100 & 264.878 & 1.854 & 0.767 \\
\hline 2 & 1239.357 & 600.002 & 0.100 & 263.152 & 1.868 & 0.767 \\
\hline 3 & 1265.237 & 600.018 & 0.100 & 266.154 & 1.844 & 0.767 \\
\hline 4 & 1241.538 & 609.339 & 0.100 & 263.515 & 1.867 & 0.767 \\
\hline 5 & 1206.238 & 600.024 & 0.100 & 259.285 & 1.900 & 0.767 \\
\hline 6 & 1185.882 & 600.117 & 0.100 & 256.763 & 1.920 & 0.767 \\
\hline
\end{tabular}


Confirmation tests were performed on these optimal cutting conditions as listed in Table 10. Three repetitions were performed for each of the six (6) experiments and the results were compared with the predicted values from RSM model and are shown in Table 11. Results show that the predicted and experimental outcomes are in conformity with one another.

Table 11: Comparison between Predicted and Experimental Results

\begin{tabular}{|c|c|c|c|c|c|c|c|c|c|}
\hline \multirow{2}{*}{ No. } & \multirow{2}{*}{$\begin{array}{c}\text { Spindle } \\
\text { Speed }\end{array}$} & \multirow{2}{*}{$\begin{array}{l}\text { Depth } \\
\text { of cut }\end{array}$} & \multirow{2}{*}{$\begin{array}{c}\text { Feed } \\
\text { rate }\end{array}$} & \multicolumn{2}{|c|}{ Residual Stress (MPa) } & \multirow{2}{*}{$\begin{array}{c}\text { Error } \\
(\%)\end{array}$} & \multicolumn{2}{|c|}{ Surface Roughness $(\mu \mathrm{m})$} & \multirow{2}{*}{$\begin{array}{c}\text { Error } \\
(\%)\end{array}$} \\
\hline & & & & Predicted & Experimental & & Predicted & Experimental & \\
\hline 1 & 1254.173 & 0.100 & 600.006 & 264.878 & 259 & 1.46 & 1.854 & 1.83 & 1.29 \\
\hline 2 & 1239.357 & 0.100 & 600.002 & 263.152 & 252 & 4.23 & 1.868 & 1.84 & 1.49 \\
\hline 3 & 1265.237 & 0.100 & 600.018 & 266.154 & 260 & 2.31 & 1.844 & 1.79 & 2.92 \\
\hline 4 & 1241.538 & 0.100 & 600.339 & 263.515 & 254 & 3.61 & 1.867 & 1.82 & 2.51 \\
\hline 5 & 1206.238 & 0.100 & 600.024 & 259.285 & 248 & 4.35 & 1.900 & 1.85 & 2.63 \\
\hline 6 & 1185.882 & 0.100 & 600.117 & 256.763 & 243 & 5.36 & 1.920 & 1.89 & 1.56 \\
\hline
\end{tabular}

\section{CONCLUSIONS}

This study deals with the experiment in which Response Surface Methodology (RSM) is used to investigate the effect of factors (Spindle speed, depth of cut and feed rate) on surface roughness and residual stress of EN24 material in an endmilling process. The experiment was performed at different spindle speed (500 - 3500 RPM), feed rates (600 - 3000 $\mathrm{mm} / \mathrm{min}$ ) and depth of cuts $(0.1-0.5 \mathrm{~mm}$ ). Analysis of Variance (ANOVA) and RSM plots were used to study the effect of factors at an individual level as well as their interactions on the response. Mathematical model was developed to contemplate the relation between input and output. Numerical optimization was performed in order to obtain optimal combination of factors satisfying the desirability criteria for response. Desirability of 0.767 showed the combination of minimum surface roughness and residual stress is obtained at minimum feed rate, minimum depth of cut and medium spindle speed. Confirmation experiments were performed at these values and it showed a good agreement between experimental and predicted values. Best optimal factor value is found to be at Spindle speed of $1254.173 \mathrm{RPM}$, feed rate of $600.006 \mathrm{~mm} / \mathrm{min}$ and depth of cut of $0.1 \mathrm{~mm}$. The obtained values of surface roughness and residual stress are $1.854 \mu$ mand $264.878 \mathrm{MPa}$.

\section{REFERENCES}

1. Tseng, Bill \& Konada, Udayvarun \& Kwon, James. (2016). A novel approach to predict surface roughness in machining operations using fuzzy set theory. Journal of Computational Design and Engineering. 3. 1-13. 10.1016/j.jcde.2015.04.002.

2. Nik Pa, Nik Masmiati \& Sarhan, Ahmed. (2015). Optimizing cutting parameters in inclined end milling for minimum surface residual stress - Taguchi approach. Measurement. 60. 267-275.

3. Bhattacharya, Anirban\& Das, Santanu \&Majumder, P. \& Batish, Ajay. (2009). Estimating the effect of cutting parameters on surface finish and power consumption during high speed machining of AISI 1045 steel using Taguchi design and ANOVA. Prod. Eng. Res. Devel.. 3. 31-40. 10.1007/s11740-008-0132-2.

4. Özel, Tuğrul\& Hsu, Tsu-Kong \& Zeren, Erol. (2005). Effects of cutting edge geometry, work piece hardness, feed raterate and cutting speed on surface roughness and forces in finish turning of hardened AISI H13 steel. The International Journal of Advanced Manufacturing Technology. 25. 262-269. 10.1007/s00170-003-1878-5.

5. Jafarian, F., Amirabadi, H. \& Fattahi, M. Improving surface integrity in finish machining of Inconel 718 alloy using intelligent systems. Int J AdvManufTechnol 71, 817-827 (2014)

6. Saikumar, S. \& Shunmugam, M. s. (2012). Investigations into high-speed rough and finish end-milling of hardened EN24 steel 
for implementation of control strategies. The International Journal of Advanced Manufacturing Technology. 63. 10.1007/s00170-012-3915-8.

7. Gupta, Munish \& Singh, Gurraj \& Sood, P.. (2015). Modelling and Optimization of Tool Wear in Machining of EN24 Steel Using Taguchi Approach. Journal of The Institution of Engineers (India): Series C. 96. 10.1007/s40032-015-0175-z.

8. ASM handbook (2005) Ultra strength steels. ASM Int I:709-715

9. Mozammel Mia, Mathematical modeling and optimization of MQL assisted end milling characteristics based on RSM and Taguchi method, Measurement, Volume 121, 2018, Pages 249-260, ISSN 0263-2241

10. Chetan Darshan, Paprinder Singh, Sanjeev Saini \& Rajat Goswami, "Comparative Evaluation of Untextured and Textured WC Inserts Under Dry and Near Dry Machining of C45 Steel”, International Journal of General Engineering and Technology (IJGET), Vol. 6, Issue 5, pp. 1-16

11. Parveen Saini \& Suraj Choudhary, “Analysis of Machining Parameters for the Optimization of Surface Roughness of Stainless Steel AISI 202 in CNC Face Milling Process", International Journal of Mechanical Engineering (IJME), Vol. 2, Issue 3, pp. 2734

12. Bishnu M Jha \& A. Mandal, "Neural Network Based Models for Surface Roughness Obtained During Electrochemical Machining of SG Iron”, International Journal of Mechanical Engineering (IJME), Vol. 5, Issue 5, pp. 7-18

13. Rajendrsinh Darbar \& Prajesh M. Patel, "Optimization of Fused Deposition Modeling Process Parameter for Better Mechanical Strength and Surface Roughness", International Journal of Mechanical Engineering (IJME), Vol. 6, Issue 6, pp. 7 - 18 